hep-th/9412033

TUW-94/20

ITP-UH-16/94

\title{
Landau-Ginzburg orbifolds with discrete torsion
}

\author{
Maximilian KREUZER同 \\ Institut für Theoretische Physik, Technische Universität Wien \\ Wiedner Hauptstraße 8-10, A-1040 Wien, AUSTRIA \\ and \\ Harald SKARKE\# \\ Institut für Theoretische Physik, Universität Hannover \\ Appelstraße 2, D-30167 Hannover, GERMANY
}

\begin{abstract}
We complete the classification of $(2,2)$ vacua that can be constructed from LandauGinzburg models by abelian twists with arbitrary discrete torsions. Compared to the case without torsion the number of new spectra is surprisingly small. In contrast to a popular expectation mirror symmetry does not seem to be related to discrete torsion (at least not in the present compactification framework): The Berglund-Hübsch construction naturally extends to orbifolds with torsion; for more general potentials, on the other hand, the new spectra neither have nor provide mirror partners in our class of models.
\end{abstract}

ITP-UH-16/94

TUW-94/20

November 1994

*e-mail: kreuzer@tph.tuwien.ac.at

\# e-mail: skarke@kastor.itp.uni-hannover.de 


\section{Introduction}

Much of the early string phenomenology was based on a few examples of 4-dimensional string constructions. Only more recently there was some effort to make theoretical and phenomenological statements on the basis of explicit classifications. In a pioneering work Schellekens and Yankielowicz [1, 2] generated huge lists of string vacua that can be constructed with nondiagonal modular invariants of tensor products of minimal models. Although their approach was to perform a statistical search, their results are fairly complete at least for $(2,2)$ models, and probably also for the $(2,1)$ case. The cases of free fermions and free bosons, which appear to be quite attractive from a phenomenological point of view [3, 4, seem to be far less tractable as far as classification is concerned and the investigations were mainly limited to single twists [5, 6].

The constructive classification can be carried through very far for Landau-Ginzburg orbifolds, which contain Gepner's models as a small subclass. The authors of ref. [7] already constructed a large number of Calabi-Yau hypersurfaces in weighted projective space. Although no strict limits on the degrees and on the singularity types were known at that time, the majority of all models in this class was found. This construction was completed and extended to Landau-Ginzburg models [8] in refs. [9, 10].

In [11] we computed all abelian orbifolds [12,13 of such models without any restrictions on the twists except for the $(2,2)$ condition. In that work, however, we discarded the possibility of introducing non-trivial phases, the so-called discrete torsions [14], among the generators of the twist group. When one tries to include such phases, two major problems occur: First, the (naïve) number of possibilities is so enormous in some cases that the redundancies due to permutation symmetries of the factors in a tensor product have to be eliminated or at least reduced. Second, we need some new efficient algorithms for the computation of spectra in the presence of torsion. This problem arises for general singularity structures, where an explicit basis of the chiral ring would have to be constructed on a case by case basis, so that the projection approach is hardly managable (see below).

Fortunately the two problems do not occur simultaneously: Large permutation symmetries arise mainly from Landau-Ginzburg potentials with a simple singularity structure. Therefore we first considered the case of ADE models, i.e. tensor products of minimal models, for which we completed the classification of $(2,2)$ models in ref. [15]. In that class all diagonal abelian symmetries happen to be generated by simple currents. So the orbifolds with torsions coincide with the $N=2$ minimal models with simple current modular invariants [16] (the exceptional invariant $E_{7}$ has to be taken into account explicitly in both frameworks). Indeed, we reproduced all spectra of ref. [1] and found the 4 missing ones.]

In the present note we complete the enumeration of abelian $(2,2)$ Landau-Ginzburg orbifolds. The results for the spectra are not too interesting from a practical point of view, since the eventual breaking of $E_{6}$ will in general change the numbers of generations $[4$. Nevertheless, most of the effort in the literature has been focused on the $(2,2)$ case, which allows for general comparisons of constructions and investigations on theoretical subjects like mirror symmetry [17, 17, 18, 19].

\footnotetext{
${ }^{1}$ Schellekens then found the additional spectra by changing the statistics in their original program.
} 
In section 2 we review the construction of Landau-Ginzburg orbifolds and derive constraints on the general structure of spectra that we have to expect. These allow us to reconstruct the complete Hodge diamond from a few quantities that can be calculated efficiently for an arbitrary singularity type. In section 3 we describe the algorithms that we use for our computations and our strategies to avoid redundancies. At last we present our results and discuss some implications and prospects of further investigations.

\section{Landau-Ginzburg orbifolds and spectra}

The Landau-Ginzburg description of (super) conformal field theories relies on the assumption that, for a given lagrangian, there exists a fixed point of the renormalization group flow at which the quantum field theory is conformally invariant. In case of $N=2$ supersymmetry the superpotential is expected not to be renormalized. Therefore, for a quasi-homogeneous superpotential $W\left(X_{i}\right)$, the chiral ring turns out to be isomorphic to the local algebra of the singularity $\mathbb{C}\left[X_{i}\right] /\left\langle\partial_{i} W\right\rangle$, i.e. the quotient of the polynomial ring by the ideal generated by the gradients (which become descendant fields via their equations of motion). For a string vacuum with space-time supersymmetry we project to integral $U(1)$ charges, and we need to include the twisted sectors in order to respect modular invariance. It has been shown by Vafa [12 that the invariant states have integral charges in all sectors if the central charge $c=3 D$ is a multiple of 3.

Of course we can consider more general orbifolds. For a $(2,0)$ model the projector $j=$ $\exp \left(2 \pi i J_{0}\right)$ to integral left charges has to be contained in the twist group and the left-moving spectral flow operator, which arises as the ground state in the sector twisted by $j^{-1}$, should be invariant. In ref. [13] the left and right charges

$$
Q_{ \pm}|h\rangle=\sum_{\theta_{i}^{h} \notin \mathbf{Z}}\left(\left(\frac{1}{2}-q_{i}\right) \pm\left(\theta_{i}^{h}-\frac{1}{2}\right)\right)|h\rangle
$$

and the transformation properties under group elements $g$

$$
g|h\rangle=(-1)^{K_{g} K_{h}} \varepsilon(g, h) \frac{\operatorname{det} g_{\mid h}}{\operatorname{det} g}|h\rangle
$$

of the ground state $|h\rangle$ in the sector twisted by $h$ were derived. In these formulas $Q_{ \pm}$are the eigenvalues of $J_{0}$ and $\bar{J}_{0}$. The commuting group elements $g$ and $h$ are assumed to act diagonally. $h$ acts on the superfield $X_{i}$ by multiplication with a phase factor $\exp \left(2 \pi i \theta_{i}^{h}\right)$, and $\operatorname{det} g_{\left.\right|_{h}}$ is the determinant of the representation of $g$ restricted to the fields that are invariant under $h$. The discrete torsions $\$ 2(g, h)$ and the signs $(-)^{K_{g}}$ parametrize the phase choices of the action of $g$ in the sector $h$ that are allowed by modular invariance; note that the group actions in the Ramond sector are fixed by those in the NS sector only up to the signs $(-)^{K_{g}}$ which we are, a priory, free to choose. The formulas (1,2) refer to the NS sector; the corresponding results in the Ramond sector are obtained by spectral flow.

For a $(2,0)$ vacuum we require

$$
\varepsilon(j, g)=(-1)^{K_{g} K_{j}} \operatorname{det} g
$$

\footnotetext{
${ }^{2}$ As usual, $\varepsilon(g, h)$ have to be multiplicative in both entries and must fulfill $\varepsilon(g, h) \varepsilon(h, g)=\varepsilon(1, g)=1$.
} 
to ensure that $\left|j^{-1}\right\rangle$ (corresponding to the holomorphic three-form in the Calabi-Yau context) is invariant [13]. In fact, such vacua have $(2,1)$ supersymmetry since the alignment of $\mathrm{R}$ and NS states in tensor products is implicit in the Landau-Ginzburg framework: In the sum over spin structures the fermionic components of all superfields $X_{i}$ are understood to have identical periodicity properties; $(2,0)$ vacua could, however, be obtained by an explicit tensoring of building blocks that are described by Landau-Ginzburg orbifolds. Since we need the leftmoving supersymmetry, modular invariance strongly constrains such a construction.

For $(2,2)$ supersymmetry we also need the right-handed analogue of (3), which implies $(-1)^{K_{g}}=\operatorname{det} g$, so that all twists must have real determinants \pm 1 . Then the group transformations take the form

$$
g|h\rangle=(-1)^{K_{g}\left(K_{h}-1\right)} \varepsilon(g, h) \operatorname{det} g_{\left.\right|_{h}}|h\rangle
$$

and, since $\operatorname{det} j=\exp \left(2 \pi i \sum q_{i}\right)$ and $D=\sum 1-2 q_{i}$,

$$
\varepsilon(j, g)=(-1)^{K_{g}(N-D+1)},
$$

where $N$ is the number of basic superfields $X_{1}, \ldots, X_{N}$.

Let us denote by $p_{i j}$ the number of states with left and right $U(1)$ charges $\left(q_{L}, q_{R}\right)=(i, j)$. The untwisted sector only contributes to states with $q_{L}=q_{R}$. For $(2,2)$ vacua there is the Poincaré duality $p_{i, j}=p_{D-i, D-j}$ : Using spectral flow, this can be understood as a consequence of charge conjugation in the Ramond sector [8]. The explicit mapping of states takes the following form: For any monomial $M\left(X_{i}\right)$ there exists a monomial $\bar{M}\left(X_{i}\right)$ such that $M \bar{M}$ is the unique monomial of highest weight. As that monomial is invariant under transformations with det $= \pm 1, M$ and $\bar{M}$ must transform with complex conjugate phases under any such linear symmetry. This duality is now easily extended to the full orbifold: Observe that the action of $g$ on $|h\rangle$ and the action of $g^{-1}$ on $\left|h^{-1}\right\rangle$, as given by eq. (4), are the same up to a factor $\left(\operatorname{det} g_{\left.\right|_{h}}\right)^{2}$. This factor is exactly cancelled by the phase difference between the actions of $g$ on $M_{h}$ and of $g^{-1}$ on $\bar{M}_{h}$, where $M_{h}$ and $\bar{M}_{h}$ are monomials of $h$-invariant fields such that their product is the highest weight monomial of the $h$-invariant Landau-Ginzburg model. Furthermore, the sums of the charges of $|h\rangle$ and $\left|h^{-1}\right\rangle$ are $Q_{ \pm}=\left(c-c_{h}\right) / 3$, where $c_{h}$ is the contribution of the untwisted fields to the central charge. Hence, if $M_{h}|h\rangle$ contributes to $p_{i j}$ then $\bar{M}_{h}\left|h^{-1}\right\rangle$ contributes to $p_{D-i, D-j}$.

We get further restrictions if $i=0$ or $j=0$. Consider, for example, states with vanishing left charge $Q_{+}=0$. We have shown in [9] that such a state must be a twisted ground state $\left|j_{a}\right\rangle$, where the group element $j_{a}$ vanishes on a subset of the fields and acts like $j$ on the remaining fields. Furthermore, the contribution of the invariant fields to the central charge must be $3\left(D-Q_{-}\right)$, where $Q_{-}$is the right charge of $\left|j_{a}\right\rangle$. It is easy to see that invariance of $\left|j_{a}\right\rangle$ under all elements of the centralizer of $j_{a}$ implies invariance of $\left|j / j_{a}\right\rangle$. Hence $p_{0 j}=p_{0, D-j}$, and by a similar reasoning $p_{i, 0}=p_{D-i, 0}$. For $D=3$ the same result can be obtained for a

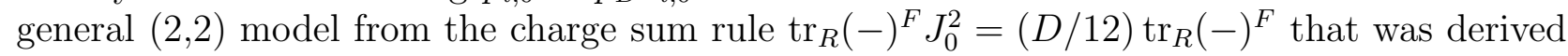
in [20]. Equality of

$$
\frac{1}{2} \operatorname{tr}(-)^{F}=p_{20}-p_{10}+p_{02}-p_{01}+p_{11}-p_{21}
$$

and

$$
2 \operatorname{tr}(-)^{F}\left(J_{0}\right)^{2}=p_{20}-p_{10}+9\left(p_{02}-p_{01}\right)+p_{11}-p_{21}
$$

implies $p_{01}=p_{02}$; the same consideration for the right moving charges implies $p_{10}=p_{20}$. 
For $p_{01}$ we can also derive the upper limit $D$. To arrive at this result we show that if the two twists $j_{a}$ and $j_{b}$ contribute to $p_{01}$ this implies that $j_{a}$ and $j_{b}$ cannot both act on the same non-trivial field (or must be identical): Let $\mathcal{M}$ be the set of fields on which both $j_{a}$ and $j_{b}$ act with a non-trivial phase, i.e. $j_{a} X_{i}=j_{b} X_{i}=j X_{i}$ for $X_{i} \in \mathcal{M}$. Invariance of $\left|j_{b}\right\rangle$ and $\left|j_{a}\right\rangle$ under all twists implies

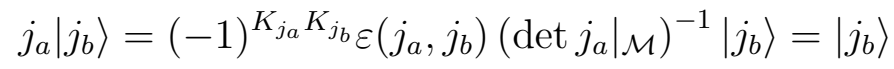

and the analogous equation with $j_{a}$ and $j_{b}$ exchanged. From $\left.\operatorname{det} j_{a}\right|_{\mathcal{M}}=\left.\operatorname{det} j_{b}\right|_{\mathcal{M}}$ we conclude that $\varepsilon\left(j_{a}, j_{b}\right)= \pm 1$ and thus $\left.\operatorname{det} j_{a}\right|_{\mathcal{M}}= \pm 1$. Finally, there are only three non-degenerate configurations with $c=3$, namely $\mathbb{C}_{(111)}[3], \mathbb{C}_{(12)}[6]$ and $\mathbb{C}_{(11)}[4]$, and the determinants of the actions restricted to $\mathcal{M}$ can only be real if $\mathcal{M}$ is empty or if $j_{a}=j_{b}$. This proves the above statement. As a corollary we can also show that $p_{01}$ can never be $D-1$ : If the states $\left|j_{a}\right\rangle$ with $1 \leq a \leq D-1$ contribute to $p_{01}$, then $\left|j_{D}\right\rangle=\left|j /\left(j_{1} \ldots j_{D-1}\right)\right\rangle$ also contributes.

Let us sum up our results on the form of the "Hodge diamond" for $D=3$. Poincaré duality $p_{i, j}=p_{D-i, D-j}$ and the results $p_{0, i}=p_{0, D-i}$ and $p_{i, 0}=p_{D-i, 0}$ derived above imply $p_{11}=p_{22}$, $p_{12}=p_{21}$ and $p_{33}=p_{30}=p_{03}=p_{00}=1$. If one of the numbers $p_{01}=p_{02}=p_{31}=p_{32}$ or $p_{10}=p_{20}=p_{13}=p_{23}$, which can only assume the values 0,1 or 3 , is different from zero, either gauge symmetry or space-time supersymmetry is enhanced. In this case the possibility of having chiral fermion generations is excluded, implying $p_{11}=p_{12}$.

In ref. [13 the possibility of twisting by group actions with negative determinant in case of odd order $d$ of $j$ was excluded because of the constraints on the discrete torsions with $j$ : Consider the case of odd $D$ and $d$, implying that $N$ is also odd, $N=2 n-1$. Taking the $d^{\text {th }}$ power of equation (5), we see that we are actually restricted to $\operatorname{det} g=1$. This seems strange, however, because by adding a trivial variable $X_{2 n}$ we can make $j$ even and thus get rid of this restriction. In fact, even without resorting to trivial fields, it is easy to see that, for odd $D$, the fields in the Ramond sector are quantized in units of $1 / 2 d$ rather that in units of $1 / d$. Thus $j$ is of order $2 d$ rather than of order $d$, and a negative determinant need not be excluded a priory if we double the range for twists by powers of $j$. We do, however, agree with the conclusion of [13] in the following sense: Modding by such symmetries can be disregarded, because the resulting orbifolds cannot yield anything new. This can be seen explicitly (at least at the level of particle spectra) from the following calculation: After adding a trivial field $X_{2 n}$, the new $j$ decomposes as $j_{\text {odd }} j_{2}$, where $j_{\text {odd }}$ is our original $j$ and $j_{2}$ acts only on $X_{2 n}$. Then we have

$$
\begin{aligned}
g\left|h j_{2}\right\rangle & =(-1)^{K_{g}\left(K_{h j_{2}}-1\right)} \varepsilon\left(g, h j_{2}\right) \operatorname{det} g_{\left.\right|_{h j_{2}}}\left|h j_{2}\right\rangle \\
& =(-1)^{K_{g} K_{h}} \varepsilon(g, h) \operatorname{det} g_{\left.\right|_{h j_{2}}}\left|h j_{2}\right\rangle
\end{aligned}
$$

and

$$
j_{2}|h\rangle=(-1)^{K_{h}-1} \operatorname{det} j_{\left.2\right|_{h}}|h\rangle .
$$

We first consider groups generated by $j_{2}$ and by elements $g$ with $\operatorname{det} g=1$ which do not act on $X_{2 n}$. Such a $g$ acts on $\left|h j_{2}\right\rangle$ in exactly the same way as it acts on $|h\rangle$, whereas the action of $j_{2}$ on any state is trivial. This means that effectively all states have been doubled by the introduction of $X_{2 n}$. Let us examine now what happens if we allow arbitrary group actions with det $= \pm 1$. It is easy to see that we can choose the group to be generated by elements $g$ as above, by $j_{2}$ and by some generator $s$ with $\operatorname{det} s=1$ and $s X_{2 n}=-X_{2 n}$, i.e. $\operatorname{det} s_{\left\{X_{1}, \ldots X_{2 n-1}\right\}}=-1$. Then

$$
s\left|h j_{2}\right\rangle=\varepsilon(s, h) \operatorname{det} s_{\left.\right|_{j_{2}}}\left|h j_{2}\right\rangle=-\varepsilon(s, h) \operatorname{det} s_{\mid h}\left|h j_{2}\right\rangle,
$$


i.e. the phase with which $s$ acts on an $h j_{2}$-twisted state is minus the phase with which it acts on the corresponding $h$-twisted state. If an $h$-twisted state survives projections by all generators with det $\left.\right|_{\left\{X_{1}, \ldots X_{2 n-1}\right\}}=1$ (in particular with $s^{2}$ ), then the action of $s$ on this state must be \pm 1 and exactly one of the two states ( $h$ - or $h j_{2}$-twisted) will survive the $s$ projection. Now let $h$ be a group element with $\operatorname{det} h_{\left\{X_{1}, \ldots x_{2 n-1}\right\}}=1$. Then

$$
j_{2}|s h\rangle=(-1)^{K_{s}+K_{h}-1} \operatorname{det} j_{\left.2\right|_{s h}}|s h\rangle=(-1)^{K_{h}-1}(-1)^{K_{h}}=-|s h\rangle,
$$

implying that no sh-twisted state can survive the $j_{2}$ projection. Therefore the states of this model are in one to one correspondence with the states of the model without $X_{2 n}$, with the group actions restricted to those with unit determinant.

\section{Computing spectra and reducing redundancies}

For models with a simple structure of the chiral ring (e.g., the case of ADE models [15]) it is quite straightforward to implement formulas (11.2) in a computer program. An alternative is provided by the formulas [13] (for a discussion adequate to the present context, see [11])

$$
\bar{\chi}=\frac{1}{|G|} \sum_{g h=h g}(-1)^{N_{h}+K_{g} K_{h}+K_{g}} \varepsilon(g, h) \prod_{\theta_{i}^{g}=\theta_{i}^{h}=0} \frac{n_{i}-d}{n_{i}},
$$

where $N_{h}$ denotes the number of $X_{i}$ invariant under $h$, and

$$
-\chi=\frac{1}{|G|} \sum_{g h=h g}(-1)^{N+K_{g} K_{h}+K_{g h}} \varepsilon(g, h) \prod_{\theta_{i}^{g}=\theta_{i}^{h}=0} \frac{n_{i}-d}{n_{i}}
$$

for the dimension of the chiral ring and Witten's index, respectively. For $D=3$ these numbers, together with $p_{10}$ and $p_{01}$, which are zero for $\chi \neq 0$, contain the full information about the spectrum.

In fact, a calculation of the Poincaré polynomial which does not require an explicit basis of the chiral ring can be performed for arbitrary Landau-Ginzburg orbifolds: The starting point is, of course, the formula

$$
P(t, \bar{t})=\prod_{i=1}^{N} \frac{1-(t \bar{t})^{1-q_{i}}}{1-(t \bar{t})^{q_{i}}}
$$

for the Poincaré polynomial of an untwisted Landau-Ginzburg model. The inverse of the denominator of this expression is the Poincaré polynomial of the freely generated polynomial ring $\mathbb{C}\left[X_{1}, \ldots, X_{N}\right]$, and the factors in the numerator correspond to dividing by the ideal that is generated by the independent polynomials $\partial_{i} W$. In a diagonal basis the action of a group element $g$ of order $\mathcal{O}(g)$ is given by $g X_{i}=\rho_{i} X_{i}$ with $\rho_{i}=\zeta^{r_{i}}$, where $\zeta$ is an $\mathcal{O}(g)^{\text {th }}$ root of unity. We can define a " $g$-extended" Poincaré polynomial

$$
P(t, \bar{t} ; \tilde{\zeta}):=\sum_{s} \sum_{r=0}^{\mathcal{O}(g)-1} \mu(r, s) \tilde{\zeta}^{r}(t \bar{t})^{s}
$$


where $\mu(r, s)$ is the number of basis monomials of weight $s$ in the chiral ring that transform with a phase $\zeta^{r}$ under the action of $g$, and $\tilde{\zeta}$ is a formal variable subject to $\tilde{\zeta}^{\mathcal{O}(g)}=1$. Then the standard arguments that lead to formula (16) give

$$
P(t, \bar{t} ; \tilde{\zeta})=\prod_{i=1}^{N} \frac{1-\tilde{\rho}_{i}^{-1}(t \bar{t})^{1-q_{i}}}{1-\tilde{\rho}_{i}(t \bar{t})^{q_{i}}}
$$

with $\tilde{\rho}_{i}=\tilde{\zeta}^{r_{i}}$ whenever $W$ is invariant under the action of $g$. We can easily get rid of $\tilde{\zeta}$ in the numerator because of

$$
\frac{1}{1-\tilde{\zeta}^{r} x}=\frac{1+\tilde{\zeta}^{r} x+\ldots+\left(\tilde{\zeta}^{r} x\right)^{\mathcal{O}(g)-1}}{1-x^{\mathcal{O}(g)}}
$$

where we used $\tilde{\zeta}^{\mathcal{O}(g)}=1$. With this identity, (18) can be recast into

$$
P(t, \bar{t} ; \tilde{\zeta})=\sum_{r=0}^{\mathcal{O}(g)-1} \tilde{\zeta}^{r} P_{r}(t \bar{t})
$$

with rational functions $P_{r}$. Of course, non-degeneracy and $g$-invariance of $W$ imply that the $P_{r}$ must be polynomials. Now the projection to invariant states is trivial (in the $h$-twisted sector we get an additional factor $t^{Q_{+}^{(h)}} \bar{t}^{Q_{+}^{(h)}} \tilde{\zeta}^{n(h)}$ describing the charges and transformation property of the twisted vacuum, and the product in (18) is restricted to fields invariant under $h$ ). This algorithm, and its obvious extension to multiple projections, can be implemented easily in an algebraic computer program. For our purposes, however, it is too slow since it would involve extensive polynomial algebra operations.

Returning to $D=3$ and integral charges, we better use (14, 115) and avoid the use of complex numbers, which come from the discrete torsions $\varepsilon(g, h)$, in the following way: Defining

$$
\alpha_{h}(g)=(-1)^{K_{g}\left(K_{h}+1\right)} \varepsilon(g, h),
$$

we see that the $h$ twisted sector yields

$$
n_{h}=\frac{1}{|G|}(-1)^{N_{h}} \sum_{g h=h g} \alpha_{h}(g) \prod_{\theta_{i}^{g}=\theta_{i}^{h}=0} \frac{n_{i}-d}{n_{i}}
$$

states, which contribute with the sign $(-1)^{K_{h}+N-N_{h}}$ to the index (15), i.e. they increase even or odd Betti numbers depending on whether $K_{h}+N-N_{h}$ is even or odd. We can define an equivalence relation among the elements of $G$ by $g \sim h$ if and only if $h=g^{\lambda}$ with $\operatorname{gcd}(\lambda, \mathcal{O}(g))=1$. Whether an $X_{i}$ is invariant under $g$ only depends on the corresponding equivalence class $[g]$ of $g$, not on the choice of $g$ within such a class. If $\mathcal{O}(g)$ is a power of a prime number $p$, then $[g]$ is the set of all $g^{\lambda}$ for which $\lambda$ is not divisible by $p$. We notice that $\alpha_{h}\left(g_{1} g_{2}\right)=\alpha_{h}\left(g_{1}\right) \alpha_{h}\left(g_{2}\right),\left|\alpha_{h}(g)\right|=1$ and $\alpha_{h}\left(1_{G}\right)=1$, i.e. $\alpha_{h}$ is a homomorphism from $G$ into $U(1)$. Therefore $\alpha_{h}(g)$ is always a power of the $\mathcal{O}(g)^{\text {th }}$ root of unity and

$$
\begin{aligned}
& \alpha_{h}(g)+\alpha_{h}\left(g^{2}\right)+\ldots+\alpha_{h}(1)=\mathcal{O}(g) \quad \text { if } \quad \alpha_{h}(g)=1 \\
& 0 \text { otherwise. }
\end{aligned}
$$


For $\mathcal{O}(g)=p^{k}$, where $p$ is a prime number, this implies

$$
\begin{array}{clll}
\sum_{g \in[g]} \alpha_{h}(g)= & 0 & \text { if } & \alpha_{h}\left(g^{p}\right) \neq 1 \\
-p^{k-1} & \text { if } & \alpha_{h}(g) \neq 1, \alpha_{h}\left(g^{p}\right)=1 \\
p^{k}-p^{k-1} & \text { if } & \alpha_{h}(g)=1 .
\end{array}
$$

Since every $g$ has a unique decomposition into elements whose orders are powers of prime numbers and this decomposition commutes with forming equivalence classes, this gives us an algorithm which avoids not only requiring knowledge about the explicit structure of the chiral ring, but also the use of complex numbers. In practice it is simpler to substitute $\alpha_{h}$ in (22) by the average of all $\alpha_{h}$ in the same class than to replace the summations over group elements by summations over classes. In our implementation we have used a mixed strategy. Of course, as in [11], we do not calculate the product over rational numbers occurring in (22) for each pair $(g, h)$, but only once for each sets of survivors. To this end we create arrays whose labels are binary codes for the sets of survivors, where we intermediately store the contributions from the $\alpha_{h}$. The resulting algorithm is similar to the one described in [11], the major difference being the fact that the occurrence of nontrivial discrete torsions forces us to go twice over the group, because we have to keep track of both $g$ and $h$.

The implementation of these concepts in a computer program is straightforward only in principle: As in [11], one could take the list of 108759 possible skeleton graphs (for an explanation of this terminology see [9]) and generate all phase symmetries. Then one has to generate all possible torsions consistent with a given symmetry group and at last the corresponding spectra have to be determined. In practice calculation time will be enormous unless some care is taken. We use the following strategy for avoiding redundacy: In a first step we can eliminate a number of skeletons for various different reasons. As we have the complete list of spectra from ADE-type skeletons [15], there is no need to redo the calculation of these. We can also eliminate all invertible skeletons containing D-type parts because of the A-D-equivalence (this would not work for non-invertible skeletons because pointers at the D-type part might spoil the argument). In addition we can eliminate skeletons with contributions of the type

$$
X_{1}^{2} X_{2}+X_{2}^{2} X_{1}
$$

because any symmetry of such a skeleton would also be a symmetry of the same skeleton with the above expression replaced by

$$
X_{1}^{3}+X_{2}^{3}
$$

Whereas the elimination of these cases was done by the computer via a sorting routine, there were also two skeletons that we eliminated by hand:

$$
X_{1}^{3}+X_{2}^{3}+X_{3}^{3}+X_{4}^{3}+X_{5}^{3}+X_{6}^{3}+X_{7}^{3}+X_{8}^{2} X_{7}+X_{9}^{2} X_{7}
$$

is redundant because all of its allowed symmetries can also be realised by skeletons of the types

$$
X_{1}^{3}+X_{2}^{3}+X_{3}^{3}+X_{4}^{3}+X_{5}^{3}+X_{6}^{3}+X_{7}^{3}+X_{8}^{2} X_{7}+X_{9}^{2} X_{8}
$$

or

$$
X_{1}^{3}+X_{2}^{3}+X_{3}^{3}+X_{4}^{3}+X_{5}^{3}+X_{6}^{3}+X_{7}^{3}+X_{8}^{2} X_{7}+X_{9}^{2} X_{6},
$$




\begin{tabular}{||rr|r||rr|r||rr|r||rr|r||rr|r||rr|r||}
\hline \hline$p_{11}$ & $p_{12}$ & $\chi$ & $p_{11}$ & $p_{12}$ & $\chi$ & $p_{11}$ & $p_{12}$ & $\chi$ & $p_{11}$ & $p_{12}$ & $\chi$ & $p_{11}$ & $p_{12}$ & $\chi$ & $p_{11}$ & $p_{12}$ & $\chi$ \\
\hline 48 & 0 & -96 & 26 & 2 & -48 & 13 & 7 & -12 & 8 & 8 & 0 & 7 & 15 & 16 & 0 & 30 & 60 \\
42 & 0 & -84 & 28 & 4 & -48 & 14 & 8 & -12 & 12 & 12 & 0 & 3 & 15 & 24 & 5 & 37 & 64 \\
30 & 0 & -60 & 21 & 3 & -36 & 11 & 7 & -8 & 7 & 11 & 8 & 3 & 19 & 32 & 0 & 42 & 84 \\
31 & 1 & -60 & 15 & 3 & -24 & 13 & 9 & -8 & 9 & 13 & 8 & 0 & 24 & 48 & 0 & 48 & 96 \\
29 & 1 & -56 & 16 & 8 & -16 & 3 & 3 & 0 & 0 & 6 & 12 & 4 & 28 & 48 & 1 & 53 & 104 \\
29 & 2 & -54 & 6 & 0 & -12 & 5 & 5 & 0 & 5 & 11 & 12 & 2 & 29 & 54 & 3 & 57 & 108 \\
24 & 0 & -48 & 11 & 5 & -12 & 6 & 6 & 0 & 7 & 13 & 12 & 1 & 29 & 56 & 2 & 58 & 112 \\
\hline \hline
\end{tabular}

Table I: The 42 ADE spectra with torsion with $p_{01}=p_{10}=0$

and

$$
X_{1}^{3}+X_{2}^{3}+X_{3}^{3}+X_{4}^{3}+X_{5}^{3}+X_{6}^{3}+X_{7}^{3}+X_{8}^{4} X_{7}
$$

is redundant because all of its allowed symmetries can also be realised by

$$
X_{1}^{3}+X_{2}^{3}+X_{3}^{3}+X_{4}^{3}+X_{5}^{3}+X_{6}^{3}+X_{7}^{3}+X_{8}^{6} .
$$

For the remaining skeletons it is necessary to decide which method for calculating spectra we want to use. Whereas the calculation of $\chi$ and $\bar{\chi}$ without explicit knowledge of the chiral ring is straightforward to implement, it has the disadvantage of being very slow. We therefore used a mixed strategy: For the two skeletons with the largest numbers of models, namely

$$
X_{1}^{3}+X_{2}^{3}+X_{3}^{3}+X_{4}^{3}+X_{5}^{3}+X_{6}^{3}+X_{7}^{2} X_{9}+X_{8}^{2} X_{7}+X_{9}^{2} X_{8}
$$

(giving rise to 365120 models) and

$$
X_{1}^{3}+X_{2}^{3}+X_{3}^{3}+X_{4}^{3}+X_{5}^{3}+X_{6}^{3}+X_{7}^{4}+X_{8}^{3} X_{7}
$$

(183680 models), it is easy to find an explicit basis for the chiral ring, so we used formulas (11:2). In all other cases, which give rise to more than one million models, we used the formulas for $\chi$ and $\bar{\chi}$. Among these other skeletons the one with the greatest number (namely 37551) of models is

$$
X_{1}\left(X_{1}^{2}+X_{2}^{2}+X_{3}^{2}+X_{4}^{2}+X_{5}^{2}+X_{6}^{2}+X_{7}^{2}+X_{8}^{2}+X_{9}^{2}\right) .
$$

This skeleton has a large permutation symmetry, but it was not necessary to take advantage of this fact because the group orders and the numbers of torsions are not too big (this is a general property of skeletons that require many additional monomials for non-degeneracy).

\section{Results and conclusions}

Our calculations result in 3937 different spectra. 2836 of these spectra come from invertible skeletons and exhibit perfect mirror symmetry, whereas 846 of the remaining 1101 spectra have no mirror in the present complete list. Comparing these numbers with the ones for Landau-Ginzburg orbifolds without torsion [11], where we had 3799] spectra (2730 of them

\footnotetext{
${ }^{3}$ In 11] we missed the spectrum $\left(p_{11}, p_{12}, \chi\right)=(30,10,-40)$, which was first found in 21], because of a programming error. Our current program reproduces that spectrum with trivial torsions.
} 


\begin{tabular}{||rr|r||rr|r||rr|r||rr|r||rr|r||rr|r||}
\hline \hline$p_{11}$ & $p_{12}$ & $\chi$ & $p_{11}$ & $p_{12}$ & $\chi$ & $p_{11}$ & $p_{12}$ & $\chi$ & $p_{11}$ & $p_{12}$ & $\chi$ & $p_{11}$ & $p_{12}$ & $\chi$ & $p_{11}$ & $p_{12}$ & $\chi$ \\
\hline 73 & 4 & -138 & 39 & 5 & -68 & 33 & 10 & -46 & 17 & 2 & -30 & 8 & 2 & -12 & 1 & 13 & 24 \\
79 & 10 & -138 & 35 & 2 & -66 & 22 & 0 & -44 & 19 & 4 & -30 & 10 & 4 & -12 & 5 & 20 & 30 \\
59 & 2 & -114 & 46 & 13 & -66 & 31 & 9 & -44 & 20 & 5 & -30 & 9 & 5 & -8 & 7 & 22 & 30 \\
58 & 4 & -108 & 34 & 4 & -60 & 35 & 13 & -44 & 23 & 8 & -30 & 12 & 8 & -8 & 8 & 23 & 30 \\
59 & 7 & -104 & 34 & 5 & -58 & 22 & 1 & -42 & 13 & 1 & -24 & 14 & 10 & -8 & 30 & 45 & 30 \\
49 & 2 & -94 & 36 & 8 & -56 & 29 & 8 & -42 & 19 & 8 & -22 & 13 & 10 & -6 & 6 & 22 & 32 \\
48 & 2 & -92 & 38 & 10 & -56 & 30 & 9 & -42 & 12 & 2 & -20 & 9 & 7 & -4 & 0 & 18 & 36 \\
45 & 4 & -82 & 44 & 16 & -56 & 22 & 2 & -40 & 16 & 6 & -20 & 20 & 22 & 4 & 2 & 20 & 36 \\
41 & 1 & -80 & 31 & 4 & -54 & 18 & 0 & -36 & 21 & 11 & -20 & 28 & 31 & 6 & 7 & 26 & 38 \\
42 & 2 & -80 & 40 & 13 & -54 & 24 & 6 & -36 & 14 & 5 & -18 & 5 & 9 & 8 & 11 & 37 & 52 \\
40 & 1 & -78 & 26 & 0 & -52 & 21 & 4 & -34 & 15 & 6 & -18 & 2 & 8 & 12 & 5 & 32 & 54 \\
42 & 3 & -78 & 28 & 2 & -52 & 17 & 1 & -32 & 10 & 2 & -16 & 13 & 20 & 14 & 13 & 40 & 54 \\
43 & 4 & -78 & 29 & 3 & -52 & 20 & 4 & -32 & 14 & 6 & -16 & 5 & 13 & 16 & 4 & 43 & 78 \\
44 & 5 & -78 & 30 & 4 & -52 & 22 & 6 & -32 & 17 & 9 & -16 & 9 & 18 & 18 & 4 & 56 & 104 \\
43 & 5 & -76 & 35 & 9 & -52 & & & & & & & & & & & \\
\hline \hline
\end{tabular}

Table II: The 86 new spectra that require torsion and do not occur for ADE models

came from invertible skeletons and 817 were mirrorless), we see that we have found only 138 new spectra. These are plotted in figure 1 against the background of the spectra that can be obtained without torsion. Among them there are 10 asymmetric spectra with $p_{01} \neq p_{10}$, namely $p_{11}=p_{12} \in\{6,10,12\}$ with $\left\{p_{01}, p_{10}\right\}=\{0,1\}, p_{11}=p_{12}=0$ with $\left\{p_{01}, p_{10}\right\}=\{0,3\}$ and $p_{11}=p_{12}=3$ with $\left\{p_{01}, p_{10}\right\}=\{1,3\}$, which occur already in the ADE case [15]. The remaining 42 ADE spectra that do not occur for Landau-Ginzburg orbifolds without torsion are listed in table I, whereas the 86 new non-ADE spectra are listed in table II. They all have $p_{01}=p_{10}=0$.

Focusing our attention on models with small modulus of the Euler number $|\chi|$, we observe that we found 2 new 2 -generation spectra and 2 new 3 -generation spectra. $(13,10,-6)$ is the model with the smallest dimension of the chiral ring among all 3-generation models that can be obtained as abelian orbifolds of Landau-Ginzburg-models. This model occurs at noninvertible points in the notorious configuration with 4 fields of weight $1 / 3$ and 3 fields of weight $2 / 9$, which is also the starting point for Schimmrigk's non-abelian model with the spectrum $(9,6,-6)$ [22].

Our numbers indicate that the inclusion of models with non-trivial discrete torsion does not improve mirror symmetry. This is also reflected in the fact that out of the 817 mirrorless spectra coming from theories without torsion, only 25 have naïve mirrors (i.e., models with exchanged Hodge numbers) among the models with torsion. For the models that come from invertible skeletons, on the other hand, mirror symmetry is perfect because the BerglundHübsch construction [18] generalizes to the case with torsion [19].

Although considerable care was necessary in the selection of algorithms and in the organization of the computations, quite a few reserves in improving efficiency are left. The two major remaining sources of redundancies are permutation symmetries and repetitions because of different skeletons giving rise to the same weights and symmetries. With some effort it 
should be possible to reduce these effects by a considerable amount. Hence the complete classification of $(2,1)$ vacua should be fairly straightforward and even the $(2,0)$ case could be viable. But from a phenomenological point of view, non-abelian symmetries and a further breaking of the gauge group are probably more interesting.

Acknowledgements. We would like to thank Albrecht Klemm and Alexander Niemeyer for informing us about their work on abelian orbifolds [21], where they first found the spectrum $(30,10,-40)$ that was missing in [1]. The work of M.K. is supported in part by the Österreichische Nationalbank under grant No. 5026. 


\section{References}

[1] A.N.Schellekens and S.Yankielowicz, Nucl. Phys. B330 (1990) 103;

Tables Supplements CERN-TH.5440S/89 and CERN-TH.5440T/89 (unpublished)

[2] J.Fuchs, A.Klemm, C.Scheich and M.Schmidt, Phys. Lett. B232 (1989) 317;

Ann. Phys. 204 (1990) 1

[3] A.E.Faraggi, Phys. Lett. B326 (1994) 62

[4] A.Font, L.E.Ibáñez, F.Quevedo and A.Sierra, Nucl. Phys. B337 (1990) 119

[5] S.A.Abel, C.M.A.Scheich, Phys. Lett. B312 (1993) 423; RAL-94-043 and FTUAM-10/94 preprint, hep-th/9404176

[6] L.E.Ibáñez, J.Mas, H.P.Nilles, F.Quevedo, Nucl. Phys. B301 (1988) 157;

A.Font, L.E.Ibáñez, F.Quevedo, Phys. Lett. B217 (1989) 272;

T.Mohaupt, MS-TPI-93-09 preprint

[7] P.Candelas, M.Lynker and R.Schimmrigk, Nucl. Phys. B341 (1990) 383

[8] C.Vafa and N.Warner, Phys. Lett. B218 (1989) 51;

E.Martinec, Phys. Lett. B217 (1989) 431;

W.Lerche, C.Vafa and N.P.Warner, Nucl. Phys. B324 (1989) 427

[9] M.Kreuzer and H.Skarke, Nucl. Phys. B388 (1992) 113;

Comm. Math. Phys. 150, 137 (1992)

[10] A.Klemm and R.Schimmrigk, Nucl. Phys. B411 (1994) 559

[11] M.Kreuzer and H.Skarke, Nucl. Phys. B405 (1993) 305

[12] C.Vafa, Mod. Phys. Lett. A4 (1989) 1169; Superstring Vacua, preprint HUTP-89/A057

[13] K.Intriligator and C.Vafa, Nucl. Phys. B339 (1990) 95

[14] C.Vafa, Nucl. Phys. B273 (1986) 592

[15] M.Kreuzer and H.Skarke, Phys. Lett. B318 (1993) 305

[16] M.Kreuzer and A.N.Schellekens, Nucl. Phys. B411 (1994) 97

[17] B.R.Greene and M.R.Plesser, Nucl. Phys. B338 (1990) 15

[18] P.Berglund and T.Hübsch, Nucl. Phys. B393 (1993) 377;

P.Berglund, M.Henningson, IASSNS-HEP-93-92 preprint, hep-th/9401029

[19] M.Kreuzer, Phys. Lett. B328 (1994) 312

[20] O.Aharony, S.Yankielowicz and A.N.Schellekens, Nucl. Phys. B418 (1994) 157

[21] A.Niemeyer, diploma thesis, TU-Munich, August 1993 (unpublished)

[22] R.Schimmrigk, Phys. Lett. B193 (1987) 175 


\section{Figures}

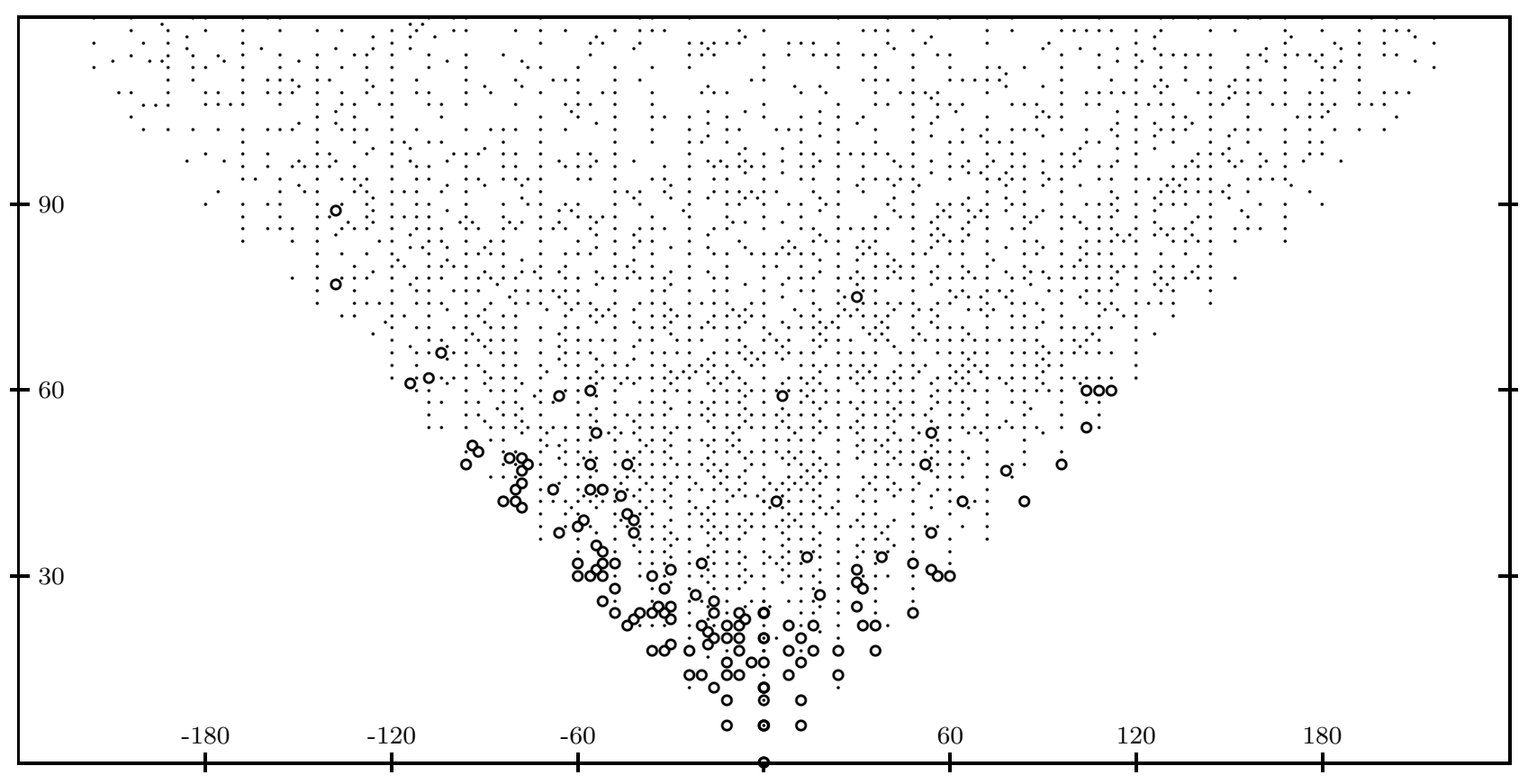

Fig. 1: $p_{11}+p_{12}$ vs. Euler number for spectra not occurring for any Landau-Ginzburg orbifold without torsion (circles), and all others with $p_{11}+p_{12} \leq 120$ (dots). 